\title{
Concurrent proton-pump inhibitors increase risk of death for lung cancer patients receiving Ist-line gefitinib treatment - a nationwide population-based study
}

This article was published in the following Dove Press journal:

Cancer Management and Research

\author{
Yu-Hung Fang' \\ Yao-Hsu Yang (iD ${ }^{2-5}$ \\ Meng-Jer Hsieh iD ${ }^{6,7}$ \\ Ming-Szu Hung ${ }^{8,9}$ \\ Yu-Ching Lin (iD) 1,8
}

'Division of Thoracic Oncology, Department of Pulmonary and Critical Care Medicine, Chang Gung Memorial Hospital, Chiayi Branch, Puzi City, Chiayi County, Taiwan, R.O.C;

${ }^{2}$ Department of Traditional Chinese Medicine, Chang Gung Memorial Hospital, Chiayi Branch, Puzi City, Chiayi County, Taiwan, R.O.C;

${ }^{3}$ Center of Excellence for Chang Gung Research Datalink, Chang Gung Memorial Hospital, Chiayi Branch, Puzi City, Chiayi County, Taiwan, R.O.C; ${ }^{4}$ Institute of

Occupational Medicine and Industrial Hygiene, National Taiwan University College of Public Health, Taipei City, Taiwan, R.O.C; ${ }^{5}$ School of Traditional Chinese Medicine, College of Medicine, Chang Gung University, Guishan Township, Taoyuan County, Taiwan, R.O.C ${ }^{6}$ Department of Respiratory Therapy, College of Medicine, Chang Gung University, Guishan Township, Taoyuan County, Taiwan, R.O.C; ${ }^{7}$ Division of Pulmonary Infection and Critical Care Medicine, Department of Pulmonary and Critical Care Medicine, Chang Gung Memorial Hospital, Chiayi Branch, Puzi City, Chiayi County, Taiwan, R.O.C; ${ }^{8}$ Department of Respiratory Care, Chang Gung University of Science and Technology, Chiayi Campus, Puzi City, Chiayi County, Taiwan, R.O.C; 'School of Medicine, College of Medicine, Chang Gung University, Guishan Township, Taoyuan County, Taiwan, R.O.C

Correspondence: Yu-Ching Lin Division of Thoracic Oncology, Department of Pulmonary and Critical Care Medicine, Chang Gung Memorial Hospital, Chiayi Branch, No.6, W. Sec., Jiapu Road, Puzi City, Chiayi County 61363, Taiwan, R.O.C

Tel +8865362 1000 Ext 2762

Fax +88653623005

Email lin0927@cgmh.org.tw
Purpose: Concurrent proton pump inhibitor (PPI) use might reduce the plasma concentration of epidermal growth factor receptor-tyrosine kinase inhibitors (EGFR-TKIs). Clinically, the adverse effect of PPIs on patients with non-small cell lung cancer (NSCLC) treated with first-line EGFR TKIs remains controversial. This study was conducted to evaluate whether the combined use of gefitinib with PPIs affected NSCLC outcomes.

Patients and methods: We performed a nationwide cohort study of patients newly diagnosed with NSCLC between 1997 and 2013 using the Taiwan Cancer Registry and Taiwan National Health Insurance databases. We identified patients who were treated with first-line EGFR TKIs and analyzed the association between use of PPIs and TKI treatment outcome. We defined the coverage ratio of PPIs as duration of PPI treatment in days divided by duration of TKIs in days. Patients who exhibited an overlap of $>20 \%$ between PPI and TKI usage days were defined as having a high coverage ratio.

Results: A total of 1278 patients were treated with first-line gefitinib, 309 of which took PPIs at the same time and 145 had a high PPI coverage ratio. Patients had similar time to failure regardless of their PPI coverage ratio during gefitinib treatment. However, higher PPI coverage ratio significantly decreased overall survival (OS) compared with that of patients with a lower PPI coverage ratio or no PPI treatment in univariate analysis (median OS, 13.5, 16.7, and 21.8 months, respectively, $p<0.01$ ) and multivariate analyses (high coverage ratio HR: 1.67; low coverage ratio HR: 1.29). Exposure to PPIs during first line gefitinib treatment significantly decreased overall survival of patients with NSCLC.

Conclusion: Concurrent use of PPIs was associated with lower overall survival in patients with EGFR-mutant NSCLC under first-line gefitinib treatment.

Keywords: proton pump inhibitor, epidermal growth factor receptor, non-small cell lung cancer, gefitinib, tyrosine kinase inhibitor, National Health Insurance Research Database

\section{Introduction}

Lung cancer is the leading cause of cancer-related mortality worldwide. ${ }^{1}$ Over the last decade, one of the most important advances for the therapy of non-small cell lung cancer (NSCLC) was the development of epidermal growth factor receptor (EGFR) tyrosine kinase inhibitors (TKIs). ${ }^{2,3}$ EGFR mutations are detected in more than $50 \%$ of patients with the histological subtype of adenocarcinoma in East Asia. ${ }^{4,5}$ Among patients harboring an activating EGFR mutation, EGFR-TKIs significantly improve not only the patient's quality of life, ${ }^{6}$ but also progression-free survival (PFS) ${ }^{7-9}$ and 
overall survival $(\mathrm{OS})^{10}$ compared with platinum-based doublet chemotherapy. Nevertheless, almost all patients who initially respond to EGFR-TKIs eventually show disease progression. Many studies have aimed to extend the effective period of EGFR-TKIs. ${ }^{11}$ However, identifying potential drug-drug interactions that may reduce the efficacy of EGFR-TKIs is equally important.

There are two widely available first-generation EGFRTKIs, gefitinib and erlotinib, and most studies have focused on erlotinib. ${ }^{12}$ The recommended dose of gefitinib (250 mg/ day) for NSCLC is around only one-third of its maximum tolerated dose, but the recommended dose used for erlotinib $\left(150 \mathrm{mg} /\right.$ day) is its maximum tolerated dose. ${ }^{13}$ Besides, compared with gefitinib, exposure to erlotinib results in both higher maximum plasma concentration (Cmax) and the area under the serum concentration versus time curve (AUC) at standard dosing. ${ }^{14}$ Therefore, we assume that the effects of gefitinib are more likely to be affected by concurrent drug treatments than the effects of erlotinib would.

Proton pump inhibitors (PPIs), the major treatment for patients with reflux esophagitis or peptic ulcers, are one of the most widely used classes of drugs in the world. In healthy volunteers, PPI use may decrease the AUC and peak plasma concentration of first-generation EGFRTKIs. ${ }^{15}$ However, the clinical impact of PPIs on the treatment outcome of EGFR-TKIs remains controversial. ${ }^{12,16-18}$ There have been few large, multi-center studies focused on patients receiving first-line EGFR-TKIs, and all the studies enrolled patients being treated with other antacid agents, not PPIs only. Therefore, we used nationwide data from the Taiwan National Health Insurance Research Database to evaluate the impact of PPIs on first-line EGFR-TKIs treatment outcomes.

\section{Materials and methods}

\section{Data source}

In Taiwan, the National Health Insurance (NHI) is a single-payer mandatory national health insurance system that covers more than $99 \%$ of citizens and provides comprehensive medical care. ${ }^{19}$ The National Health Insurance Research Database (NHIRD), established by the National Health Research Institutes (NHRI) of the Taiwanese government, is one of the largest and most complete healthcare databases in the world, and has been used to thoroughly study lung cancer. ${ }^{20}$ In the database, all information is encrypted to protect the anonymity of the patients. This study met the confidentiality regulations of the Bureau of NHI. The institutional review board of Chang Gung Memorial Hospital proved that patient consent was not required to review their medical records in this retrospective database study (201700257B1). Patients' anonymity and confidentiality were ensured throughout the study.

\section{Definition of lung cancer}

Using the NHIRD, we identified patients older than 18 years of age with lung cancer according to the 9th Revision International Classification of Disease, Clinical Modification [ICD-9-CM] code 162 from the Registry of Catastrophic Illness Patients Database. The Registry of Catastrophic Illness Patients Database is a subset of NHIRD, and pathological confirmation of lung cancer is required to apply this registry.

\section{Definition of first-line EGFR-TKI}

Among patients with ICD-9-CM code 162 between 1997 and 2013, we used Anatomical Therapeutic Chemical (ATC) code to identify patients with NSCLC who were treated with gefitinib between Jun 2011 and Jun 2013. This timeline was chosen because NHI has reimbursed EGFRTKIs as a first-line therapy for late-stage EGFR-mutant primary lung cancer since Jun 2011.

According to the NHI policy, physicians must seek approval every 3 months when prescribing first-line EGFR-TKIs with initial pathological diagnosis, EGFR mutation type analysis, and image evidence confirming advanced lung cancer in patients, and re-apply every 3 months according to the tumor response as evaluated by image studies with chest computer tomography, bone scans, and brain magnetic resonance imaging, which must be peer reviewed. NHI policy states that EGFR-TKI use is not allowed beyond radiological progression.

To exclude patients who were not taking first-line EGFR-TKIs, we identified patients who had received prior chemotherapy using ATC codes including: L01XA01 (Cisplatin); L01XA02 (Carboplatin); L01BA04 (Pemetrexed); L01CA04 (Vinorelbine); L01CD01 (Paclitaxel); L01CD02 (Docetaxel); and L01BC05 (Gemcitabine).

Thus, according to the NHI policy, patients taking firstline EGFR-TKIs without previous chemotherapy must have late-stage EGFR-mutant primary lung cancer. For those treated with first-line EGFR-TKIs, we followed from the index date of gefitinib use until treatment failure, death, or the end of 2013. Time to treatment failure (TTF) 
was defined as the time from the start of the first-line treatment to the last day of receiving gefitinib. The last prescription date was further confirmed by observing no additional prescription of gefitinib within the subsequent 28 days.

\section{Definition of PPIs}

Using ATC codes including A02BC01 (Omeprazole); A02BC03 (Lansoprazole); A02BC05 (Esomeprazole); A02BC02 (Pantoprazole); A02BC04 (Rabeprazole); A02BC06 (Dexlansoprazole), we identified patients who were prescribed PPIs after starting EGFR-TKI therapy. We defined the PPI coverage ratio as the duration of PPI treatment in days divided by the duration of TKI treatment in days. Patients who exhibited an overlap of $>20 \%$ between PPI and TKI usage days were defined as having a high coverage ratio.

\section{Statistical data analysis}

Differences between high and low PPI coverage ratios were evaluated using Student's $t$-test. TTF, OS, and 95\% confidence intervals (CIs) were compared using the Kaplan-Meier method. Comparisons between groups were performed by the log-rank test. Association of risk factors was evaluated with Cox proportional hazards regression modeling. Variables including age, gender, urbanization level, income, and comorbidities were included in the multivariable analysis. Statistical significance was defined as $p<0.05$. All analyses were performed using the statistical package SPSS version 20 (IBM SPSS Inc, Chicago, IL, USA).

\section{Results}

A total of 2021 patients with lung cancer that were diagnosed between Jun 2011 and Jun 2013 (Figure 1), older than 18 years old, and received first-line gefitinib treatment were included. We excluded 743 patients. Among them, 252 expired within 3 months after initiating gefitinib treatment (and took gefitinib for less than 3 months) $(\mathrm{n}=252) ; 567$ had previous frontline chemotherapy; 93 took gefitinib for less than 3 months and had previous frontline chemotherapy; and 17 patients had missing data (urban: $\mathrm{n}=17$ ).

There were 969 patients who took gefitinib only, and they used gefitinib for 331.9 \pm 213.5 days on average. Among 309 patients who took PPIs during their gefitinib treatment period, PPIs were used for $78.4 \pm 104.1$ days while using gefitinib for $347.1 \pm 211.8$ days on average. Among patients with

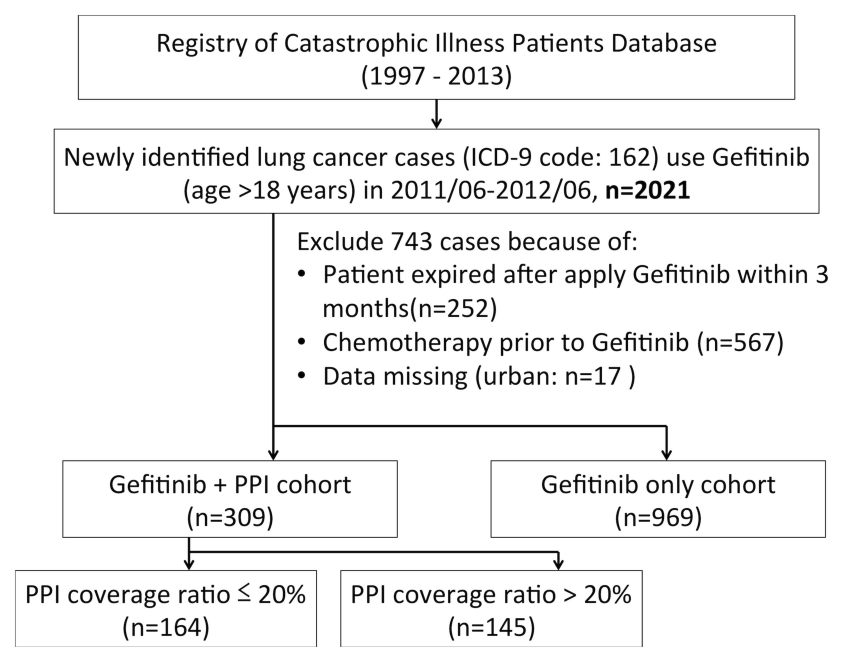

Figure I Flow of patients through the study.

combination therapy, 164 who had a PPI coverage ratio less than $20 \%$ were defined as the low coverage ratio group, and 145 patients who had a PPI coverage ratio more than $20 \%$ were defined as the high coverage ratio group (Figure 1). In patients with high and low PPI coverage ratios, PPIs were used for $142.0 \pm 120.9$ days and $22.2 \pm 28.1$ days on average, respectively. There was no statistically significant difference between patients who had combination therapy or not regarding gender, age, urbanization level, and income. Patients receiving PPI and TKI combination therapy had a higher percentage of chronic obstructive pulmonary disease than those receiving gefitinib treatment alone $(62.14 \%$ versus $31.58 \%, p=0.0409$ ) (Table 1).

As of the last follow-up date, $985(77.07 \%)$ patients exhibited gefitinib treatment failure and 632 (49.45\%) had died. The patients with single gefitinib treatment, low PPI coverage ratio, and high PPI coverage ratio had similar median TTF values (median: $0.83,0.97$, and 0.74 years, respectively, $p=0.32$, Figure 2 ). However, patients with high PPI coverage ratio had a significantly shorter OS compared to that of patients with a low PPI coverage ratio or gefitinib treatment only (median, 1.82, 1.39 and 1.12 years, respectively, $p<0.01$, Figure 3 ).

Multivariate analyses using Cox's proportional hazard model revealed no significant difference in TTF among patients with single gefitinib or combination therapy of PPIs with gefitinib, and no association between TTF and age, urbanization level, or comorbidities including diabetes, hypertension, and chronic obstructive pulmonary disease. The adjusted hazard ratios (HR) of TTF in groups with lower and higher coverage ratios compared with 
Table I Baseline patient characteristics

\begin{tabular}{|c|c|c|c|c|c|c|c|c|c|c|}
\hline \multirow[t]{2}{*}{ Variable } & \multicolumn{2}{|c|}{$\begin{array}{l}\text { High coverage ratio, } \\
N=\mid 45\end{array}$} & \multicolumn{2}{|c|}{$\begin{array}{l}\text { Low coverage ratio, } \\
N=164\end{array}$} & \multirow[t]{2}{*}{$p$-value } & \multicolumn{2}{|c|}{$\begin{array}{l}\text { Gefitinib with } \\
\text { PPIs } \\
\text { Total N=309 }\end{array}$} & \multicolumn{2}{|c|}{$\begin{array}{l}\text { Gefitinib } \\
\text { only } \\
N=969\end{array}$} & \multirow[t]{2}{*}{$p$-value } \\
\hline & $\mathbf{N}$ & (\%) & $\mathbf{N}$ & (\%) & & $\mathbf{N}$ & (\%) & $\mathbf{N}$ & (\%) & \\
\hline \multicolumn{11}{|l|}{ Gender } \\
\hline Male & 42 & 28.97 & 63 & 38.41 & 0.080 & 105 & 33.98 & 346 & 35.71 & 0.580 \\
\hline Female & 103 & 71.03 & 101 & 61.59 & & 204 & 66.02 & 623 & 64.29 & \\
\hline \multicolumn{11}{|l|}{ Age (years) } \\
\hline Young $(\leq 65)$ & 62 & 42.76 & 65 & 39.63 & 0.578 & 182 & 58.90 & 558 & 57.59 & 0.684 \\
\hline Old $(>65)$ & 83 & 57.24 & 99 & 60.37 & & 127 & 41.10 & 411 & 42.41 & \\
\hline \multicolumn{11}{|l|}{ Income (TWD) } \\
\hline 0 & 23 & 15.86 & 33 & 20.12 & 0.096 & 56 & 18.12 & 175 & 18.06 & 0.090 \\
\hline$I-15,840$ & 19 & 13.10 & 14 & 8.54 & & 33 & 10.68 & 137 & 14.14 & \\
\hline$|5,84|-25,000$ & 85 & 58.62 & 83 & 50.61 & & 168 & 54.37 & 456 & 47.06 & \\
\hline$>25,000$ & 18 & $|2.4|$ & 34 & 20.73 & & 52 & 16.83 & 201 & 20.74 & \\
\hline \multicolumn{11}{|l|}{ Comorbidities } \\
\hline DM & 47 & 32.41 & 53 & 32.32 & 0.986 & 100 & 32.36 & 259 & 26.73 & 0.055 \\
\hline Hypertension & 96 & 66.21 & 105 & 64.02 & 0.688 & 201 & 65.05 & 605 & 62.44 & 0.407 \\
\hline COPD & 54 & 37.24 & 63 & 38.41 & 0.832 & 117 & 62.14 & 306 & 31.58 & $0.04 I^{*}$ \\
\hline
\end{tabular}

Note: $* P$-value $<0.05$.

Abbreviations: PPI, proton-pump inhibitor; TWD, new Taiwan dollar; DM, diabetes mellitus; COPD, chronic obstructive pulmonary disease.

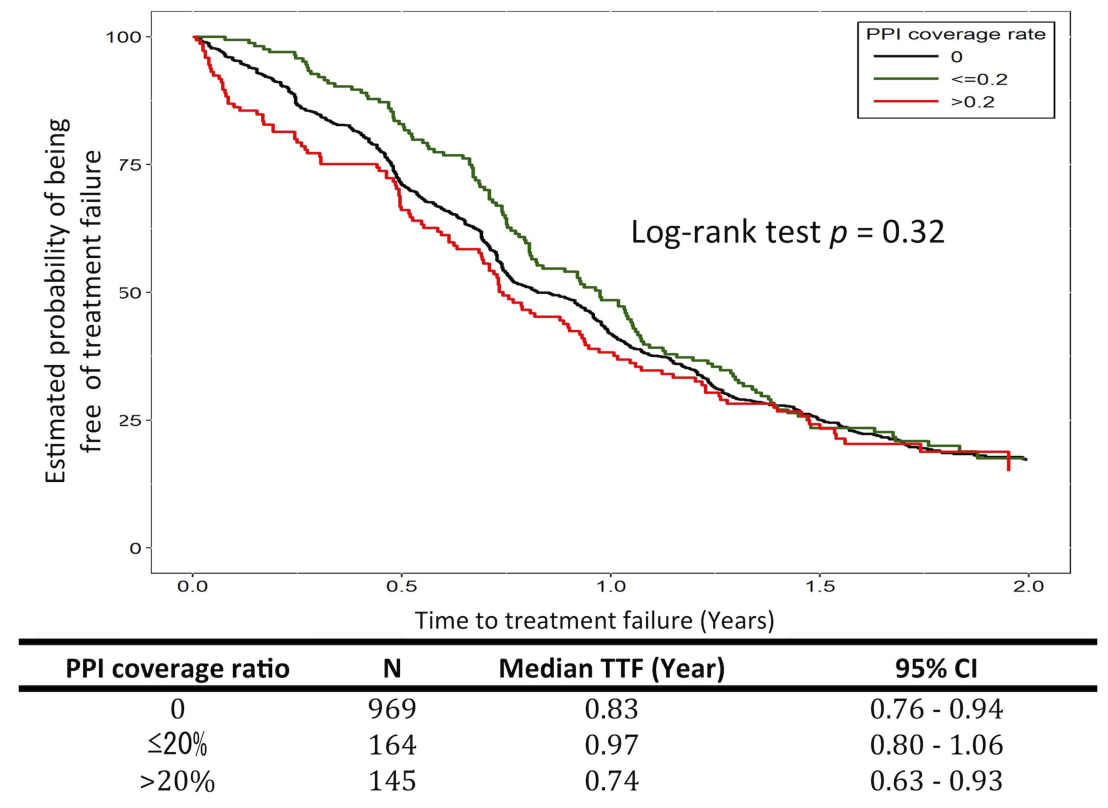

Figure 2 Time to treatment failure.

single gefitinib group were 0.89 (95\% CI: $0.74-1.08$, treatment had a better OS compared to that of patients $p=0.239)$ and $1.11 \quad(95 \%$ CI: $0.91-1.36, p=0.306)$. with low or high PPI coverage ratios (lower coverage ratio Significant associations were found between shorter TTF HR: $1.29,95 \%$ CI: $1.03-1.62, p=0.027$; higher coverage and male sex (adjusted HR $=1.15,95 \%$ CI: $1.00-1.32$, ratio HR: 1.67, 95\% CI: $1.33-2.09, p<0.001$ ) (Figure 3). $p=0.047)$ and lower monthly income $(p=0.0015)$. Other significant associations were found with male sex Additionally, patients who received single gefitinib (HR: 1.43, 95\% CI: 1.21-1.69, $p<0.001$ ), age older than 


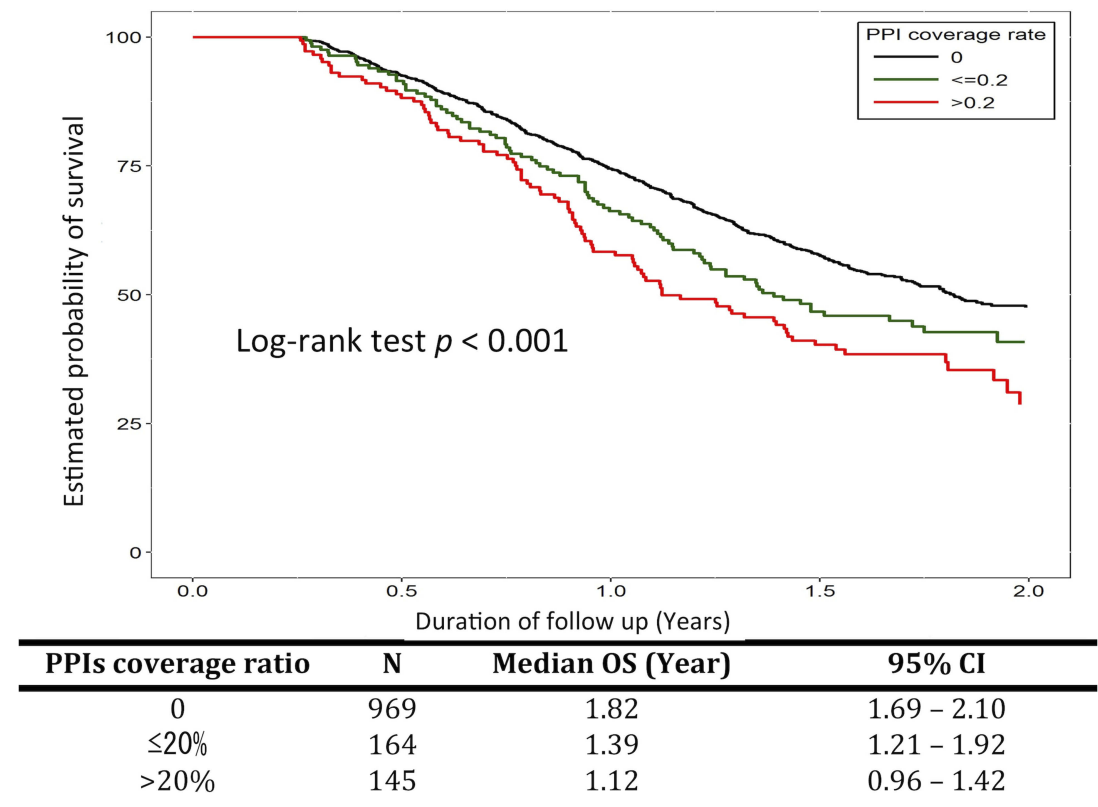

Figure 3 Overall survival.

65 (HR: 1.45, 95\% CI: 1.21-1.75, $p<0.001$ ), and lower monthly income $(p<0.001)$ (Table 2$)$.

\section{Discussion}

This nationwide population-based cohort study evaluated the impact of PPIs on first-line EGFR-TKIs among patients with NSCLC. In the present study, combined use of PPIs with EGFR-TKIs was dose-dependently associated with a significantly reduced OS. Risk of death among patients with low and high PPI coverage ratios was increased $29 \%$ and $67 \%$, respectively. This result was essentially compatible with results of previous study by Chen et al. ${ }^{18}$ Similarly, Sharma et. al recently reported that concomitant TKI-PPI treatment adversely affected OS using the SEER-Medicare database. ${ }^{21}$ Risk of mortality increased $21 \%$ within 90 days among patients with lung cancer who were receiving erlotinib with a concomitant PPI. Treatment with other TKIs like sunitinib and imatinib had no significant association between the risk of death and concomitant TKI-PPI treatment.

Despite the recommendation of the US Food and Drug Administration (FDA) to avoid concomitant use of gefitinib with PPIs, we found that $24.17 \%$ patients with lung cancer taking first-line gefitinib received PPIs at the same time. In practice, a higher percentage of patients with concomitant use of gefitinib and PPIs could be expected since the NHI only reimburses PPIs for patients with reflux esophagitis or peptic ulcers confirmed by panendoscopy or $24 \mathrm{hr}$ pH-meter monitoring within 4 months. Data for patients who may have paid for PPIs out of pocket, such as those who were unable or who declined panendoscopy, is not available in this database.

The clinical impact of combining gastric acid suppressive agents with first line TKIs among patients with EGFR-mutant NSCLC remains controversial. Hilton et al retrospectively analyzed the BR.21 trial database to evaluate the clinical outcome of gastric acid suppressive agents and erlotinib. ${ }^{12}$ There were no differences in plasma drug levels between the two groups. Gastric acid suppressive agents did not appear to negatively impact PFS or OS. On the other hand, Chu et al reported shorter PFS and OS in patients taking gastric acid suppressive agents in 507 patients with non-small cell lung cancer unselected for EGFR status. ${ }^{22}$ Multivariate Cox proportional hazards ratios for PFS and OS were 1.83 (95\% CI, 1.48-2.25) and 1.37 (95\% CI, 1.11-1.69), respectively. However, neither study selected EGFR status, and only evaluated patients receiving erlotinib. Chen et al retrospectively analyzed 269 patients receiving first-line EGFR TKIs who took antacid agents or not. ${ }^{18}$ The results showed that antacid agent use did not significantly affect PFS, but significantly reduced OS. The multivariable analysis demonstrated that the hazard ratio of OS among PPI users compared with the non-antacid agent group was 2.27 (95\% CI, 1.26-4.11). However, two other studies for first-line EGFR-TKIs both reported no significant 
Table 2 Multivariate analyses of time to treatment failure and overall survival

\begin{tabular}{|c|c|c|c|c|c|c|c|c|}
\hline \multirow[t]{3}{*}{ Variable } & \multicolumn{4}{|c|}{ Time to treatment failure } & \multicolumn{4}{|c|}{ Overall survival } \\
\hline & \multicolumn{4}{|c|}{ Adjusted } & \multicolumn{4}{|c|}{ Adjusted } \\
\hline & \multirow[t]{2}{*}{ HR } & \multicolumn{2}{|c|}{$95 \% \mathrm{Cl}$} & $P$-value & \multirow[t]{2}{*}{ HR } & \multicolumn{2}{|c|}{$95 \% \mathrm{Cl}$} & $P$-value \\
\hline Drugs & & & & & & & & \\
\hline No PPIs & 1.00 & & & & 1.00 & & & \\
\hline Low coverage ratio & 0.89 & 0.74 & 1.08 & 0.239 & 1.29 & 1.03 & 1.62 & $* 0.027$ \\
\hline High coverage ratio & I.II & 0.91 & 1.36 & 0.306 & 1.67 & 1.33 & 2.09 & $*<0.001$ \\
\hline \multicolumn{9}{|l|}{ Gender } \\
\hline Female & 1.00 & & & & 1.00 & & & \\
\hline Male & 1.15 & 1.00 & 1.32 & 0.047 & 1.43 & 1.21 & 1.69 & $*<0.001$ \\
\hline \multicolumn{9}{|l|}{ Age (years) } \\
\hline $19-65$ & 1.00 & & & & 1.00 & & & \\
\hline$>65$ & 1.02 & 0.88 & 1.18 & 0.782 & 1.45 & 1.21 & 1.75 & $*<0.001$ \\
\hline \multicolumn{9}{|l|}{ Income (TWD) } \\
\hline 0 & 1.00 & & & & 1.00 & & & \\
\hline $\mid \sim 15,840$ & 0.87 & 0.70 & 1.09 & 0.238 & 0.87 & 0.66 & 1.14 & 0.307 \\
\hline$|5,84| \sim 25,000$ & 0.83 & 0.70 & 0.98 & $* 0.032$ & 0.78 & 0.63 & 0.96 & $* 0.020$ \\
\hline$>25,000$ & 0.78 & 0.63 & 0.95 & $* 0.014$ & 0.55 & 0.42 & 0.73 & $*<0.001$ \\
\hline \multicolumn{9}{|l|}{ Comorbidities } \\
\hline Diabetes mellitus & 1.09 & 0.94 & 1.26 & 0.2599 & 1.09 & 0.91 & 1.30 & 0.3478 \\
\hline Hypertension & 1.00 & 0.86 & 1.15 & 0.9613 & 1.00 & 0.83 & 1.20 & 0.9681 \\
\hline COPD & 0.94 & 0.82 & 1.07 & 0.3487 & 0.96 & 0.81 & 1.14 & 0.6248 \\
\hline
\end{tabular}

Note: *P-value $<0.05$.

Abbreviations: $\mathrm{HR}$, hazard ratio; $\mathrm{Cl}$; confidence interval; PPI, proton-pump inhibitor; TWD, new Taiwan dollar; COPD, chronic obstructive pulmonary disease.

influence on PFS or OS despite combination use of PPIs, but both studies included small sample sizes. ${ }^{16,17}$

One of the reasons PPIs may be related with changes in OS or PFS is because PPI use reflects comorbidities such as cardiovascular diseases treated with aspirin, gastric ulcers with active bleeding, or smokers with reflux esophagitis or chronic obstructive pulmonary disease. However, in the present study, there was no statistically significant difference in multivariable analysis for comorbidities including diabetes, hypertension, and chronic obstructive pulmonary disease. Combination use of PPIs and EGFR-TKIs may also increase the side effects of both drugs. In a recent study, Cho et al showed that anti-acidsecreting agents increased gefitinib-induced hepatotoxicity about 1.5- to 1.7 -fold. ${ }^{23}$ Furthermore, PPIs inhibit gastric acid secretion and increase the intra-gastric $\mathrm{pH}$, leading to gut dysbiosis and an increased risk of enteric infection and diarrhea. ${ }^{24}$ This can partially explain the increased incidence of diarrhea and infections in patients enrolled in the BR21 study receiving gastric acid suppression medications during treatment with erlotinib. ${ }^{12}$
The other theory to explain the shorter OS, but not TTF, in the PPI combination group is that more patients may have distant metastasis and/or uncontrolled metastases because of insufficient serum levels of TKIs. Previous studies show that low TKI serum levels are still sufficient for treating EGFR mutant lung cancer. However, the cerebrospinal fluid (CSF) penetration rate of first-generation TKIs is only around $2 \% .{ }^{25,26}$ Since the co-administration of PPIs decreases the absorption of EGFR-TKIs, CSF concentration of EGFR-TKIs would likely further reduce to an insufficient level. The NHI database did not record the metastatic site of lung cancer, so further studies may be needed to identify the effect of PPIs on distant metastasis of lung cancer after TKI treatment.

There were some limitations in this study. First, we could not determine which drugs were prescribed without NHI payment. In Taiwan, PPIs are only paid for by the NHI for patients with a positive result of reflux esophagitis or peptic ulcer according to endoscopic or $24 \mathrm{hr} \mathrm{pH}$ meter results within 4 months. Patients who refused such invasive studies and bought PPIs by themselves from the local 
pharmacy could not be determined. Second, there were no available laboratory results or reports of radiographic disease during progression from the NHIRD. Thus, we did not definitely know the EGFR status or disease status, but the data was reliable because of patients who taking firstline EGFR-TKIs without previous chemotherapy must have late-stage EGFR mutant lung primary lung cancer, and physicians have to apply EGFR-TKIs with initial pathological diagnosis, EGFR mutation type, and image evidence confirming advanced stage of lung cancer. To select patients with sensitive EGFR mutations more precisely, we also excluded all patients who had expired within the first 3 months of taking EGFR-TKIs. Third, we analyzed the effect of PPIs on gefitinib treatment, but there was no available data for erlotinib or afatinib from the NHIRD. The NHI has reimbursed erlotinib treatment since April 01, 2013 and afatinib since May 01, 2014, and we did not have the data released from NHIRD after Dec 31, 2013. Finally, in the present study, we could not conclude that taking PPIs directly increases the mortality of patients receiving gefitinib. However, we showed that the high coverage ratio of combined PPI and gefitinib use was associated with reduced OS through an unknown mechanism. Nevertheless, to our knowledge, this is the first and the largest nationwide cohort study to associate PPI use with a negative effect on OS among patients with EGFRmutant NSCLC treated with first-line gefitinib.

Compared with gefitinib or erlotinib, afatinib is highly soluble throughout the physiologic $\mathrm{pH}$ range (1-7.5) and may therefore have fewer interactions with acid-reducing drugs. ${ }^{27}$ Vishwanathan et al reported two phase I, openlabel studies to assess the impact of food or omeprazole on the exposure of osimertinib. ${ }^{28}$ Co-administration with omeprazole did not affect osimertinib exposure. For patients with advanced-stage EGFR mutant lung cancer who need to use PPIs, afatinib or osimertinib may be a better choice than gefitinib. Soda intake increases the bioavailability of erlotinib during esomeprazole treatment. ${ }^{29}$ For patients receiving first-line EGFR-TKIs and PPIs, taking gefitinib or erlotinib with an acidic beverage like soda could be an alternative choice.

\section{Conclusion}

Patients with EGFR-mutant NSCLC taking gefitinib with PPIs exhibited an increased risk of death by $29 \%$ and $67 \%$ in groups with low or high coverage ratios, respectively. For patients with NSCLS receiving first-line treatment with gefitinib, concurrent use of PPIs should be avoided.

\section{Abbreviations}

EGFR, epidermal growth factor receptor; TKIs, tyrosine kinase inhibitors; PFS, progression-free survival; OS, overall survival; Cmax, maximum plasma concentration; AUC, area under the serum concentration curve versus time; PPIs, Proton Pump Inhibitors; NHI, National Health Insurance; NHIRD, National Health Insurance Research Database; NHRI, National Health Research Institutes; ICD-9-CM, International Classification of Diseases, Ninth Revision, Clinical Modification; ATC, Anatomical Therapeutic Chemical; TTF, Time to treatment failure; CI, confidence interval; HR, hazard ratio; FDA, US Food and Drug Administration.

\section{Acknowledgments}

We would like to thank the Health Information and Epidemiology Laboratory of Chang Gung Memorial Hospital, Chiayi Branch, for the comments on and assistance with data analysis. This study was supported by a grant from Chang Gung Memorial Hospital, Chiayi branch, Taiwan (CORPG6D0161). This funding body played no role in study design, analysis, and interpretation of data in this paper. This study was based on a portion of data from the National Health Insurance Research Database provided by the Bureau of National Health Insurance, Department of Health, and was managed by the National Health Insurance Research Institutes, Taiwan. The statistical results and conclusions presented in this paper do not represent those of the Bureau of National Health Insurance, Department of Health, or the National Health Insurance Research Institutes.

\section{Disclosure}

The authors report no conflicts of interest in this work.

\section{References}

1. Siegel R, Naishadham D, Jemal A. Cancer statistics, 2012. CA Cancer J Clin. 2012;62(1):10-29. doi:10.3322/caac.20138

2. Morgensztern D, Politi K, Herbst RS. EGFR mutations in non-smallcell lung cancer: find, divide, and conquer. JAMA Oncol. 2015;1 (2):146-148. doi:10.1001/jamaoncol.2014.278

3. Chan BA, Hughes BG. Targeted therapy for non-small cell lung cancer: current standards and the promise of the future. Transl Lung Cancer Res. 2015;4(1):36-54. doi:10.3978/j.issn.2218-6751.2014.05. 01

4. Midha A, Dearden S, McCormack R. EGFR mutation incidence in non-small-cell lung cancer of adenocarcinoma histology: a systematic review and global map by ethnicity (mutMapII). Am J Cancer Res. 2015;5(9):2892-2911. 
5. Zheng H, Zhang Y, Zhan Y, et al. Prognostic analysis of patients with mutant and wild-type EGFR gene lung adenocarcinoma. Cancer Manage Res. 2019;11:6139-6150. doi:10.2147/CMAR.S200126

6. Oizumi S, Kobayashi K, Inoue A, et al. Quality of life with gefitinib in patients with EGFR-mutated non-small cell lung cancer: quality of life analysis of North East Japan study group 002 trial. Oncologist. 2012;17(6):863-870. doi:10.1634/theoncologist.2011-0426

7. Zhou C, Wu YL, Chen G, et al. Erlotinib versus chemotherapy as first-line treatment for patients with advanced EGFR mutation-positive non-small-cell lung cancer (OPTIMAL, CTONG-0802): a multicentre, open-label, randomised, phase 3 study. Lancet Oncol. 2011;12 (8):735-742. doi:10.1016/S1470-2045(11)70184-X

8. Wu YL, Zhou $\mathrm{C}, \mathrm{Hu} \mathrm{CP}$, et al. Afatinib versus cisplatin plus gemcitabine for first-line treatment of Asian patients with advanced nonsmall-cell lung cancer harbouring EGFR mutations (LUX-Lung 6): an open-label, randomised phase 3 trial. Lancet Oncol. 2014;15 (2):213-222. doi:10.1016/S1470-2045(13)70604-1

9. Mok TS, Wu YL, Thongprasert S, et al. Gefitinib or carboplatinpaclitaxel in pulmonary adenocarcinoma. $N$ Engl J Med. 2009;361 (10):947-957. doi:10.1056/NEJMoa0810699

10. Yang JC, Wu YL, Schuler M, et al. Afatinib versus cisplatin-based chemotherapy for EGFR mutation-positive lung adenocarcinoma (LUX-Lung 3 and LUX-Lung 6): analysis of overall survival data from two randomised, phase 3 trials. Lancet Oncol. 2015;16(2):141151. doi:10.1016/S1470-2045(14)71173-8

11. Leung L, Mok TS, Loong H. Combining chemotherapy with epidermal growth factor receptor inhibition in advanced non-small cell lung cancer. Ther Adv Med Oncol. 2012;4(4):173-181. doi:10.1177/ 1758834012440015

12. Hilton JF, Tu D, Seymour L, Shepherd FA, Bradbury PA. An evaluation of the possible interaction of gastric acid suppressing medication and the EGFR tyrosine kinase inhibitor erlotinib. Lung Cancer. 2013;82(1):136-142. doi:10.1016/j.lungcan.2013.06.008

13. Costa DB, Nguyen KS, Cho BC, et al. Effects of erlotinib in EGFR mutated non-small cell lung cancers with resistance to gefitinib. Clin Cancer Res. 2008;14(21):7060-7067. doi:10.1158/1078-0432.CCR-081455

14. Rukazenkov Y, Speake G, Marshall G, et al. Epidermal growth factor receptor tyrosine kinase inhibitors: similar but different? Anticancer Drugs. 2009;20(10):856-866. doi:10.1097/CAD.0b013e32833034e1

15. Budha NR, Frymoyer A, Smelick GS, et al. Drug absorption interactions between oral targeted anticancer agents and PPIs: is pH-dependent solubility the Achilles heel of targeted therapy? Clin Pharmacol Ther. 2012;92(2):203-213. doi:10.1038/clpt.2012.73

16. Zenke Y, Yoh K, Matsumoto S, et al. Clinical impact of gastric acidsuppressing medication use on the efficacy of erlotinib and gefitinib in patients with advanced non-small-cell lung cancer harboring EGFR mutations. Clin Lung Cancer. 2016;17(5):412-418. doi:10.1016/j.cllc.2016.01.006

17. Miyazaki K, Sato S, Kodama T, et al. Effect of acid suppressants on the efficacy of tyrosine kinase inhibitors in patients with epidermal growth factor receptor-mutated non-small-cell lung cancer. Mol Clin Oncol. 2016;4(5):873-877. doi:10.3892/mco.2016.810
18. Chen YM, Lai CH, Chang HC, et al. Antacid use and de novo brain metastases in patients with epidermal growth factor receptor-mutant non-small cell lung cancer who were treated using first-line first-generation epidermal growth factor receptor tyrosine kinase inhibitors. PLoS One. 2016;11(2):e0149722. doi:10.1371/journal.pone.0149722

19. Wu TY, Majeed A, Kuo KN. An overview of the healthcare system in Taiwan. London J Prim Care. 2010;3(2):115-119. doi:10.1080/ 17571472.2010.11493315

20. Wang BY, Huang JY, Cheng CY, Lin CH, Ko J, Liaw YP. Lung cancer and prognosis in Taiwan: a population-based cancer registry. $J$ Thorac Oncol. 2013;8(9):1128-1135. doi:10.1097/JTO.0b013e31829ceba4

21. Sharma M, Holmes HM, Mehta HB, et al. The concomitant use of tyrosine kinase inhibitors and proton pump inhibitors: prevalence, predictors, and impact on survival and discontinuation of therapy in older adults with cancer. Cancer. 2019;125(7):1155-1162. doi:10.1002/ cncr.31917

22. Chu MP, Ghosh S, Chambers CR, et al. Gastric Acid suppression is associated with decreased erlotinib efficacy in non-small-cell lung cancer. Clin Lung Cancer. 2015;16(1):33-39. doi:10.1016/j.cllc. 2014.07.005

23. Cho S, Yee J, Kim JY, Jeong Rhie S, Gwak HS. Effects of concomitant medication use on gefitinib-induced hepatotoxicity. J Clin Pharmacol. 2018;58(2):263-268. doi:10.1002/jcph.1010

24. Rossi G, Pezzuto A, Sini C, et al. Concomitant medications during immune checkpoint blockage in cancer patients: novel insights in this emerging clinical scenario. Crit Rev Oncol Hematol. 2019;142:26-34. doi:10.1016/j.critrevonc.2019.07.005

25. Togashi Y, Masago K, Masuda S, et al. Cerebrospinal fluid concentration of gefitinib and erlotinib in patients with non-small cell lung cancer. Cancer Chemother Pharmacol. 2012;70(3):399-405. doi:10. 1007/s00280-012-1929-4

26. Deng Y, Feng W, Wu J, et al. The concentration of erlotinib in the cerebrospinal fluid of patients with brain metastasis from non-smallcell lung cancer. Mol Clin Oncol. 2014;2(1):116-120. doi:10.3892/ mco.2013.190

27. Xu ZY, Li JL. Comparative review of drug-drug interactions with epidermal growth factor receptor tyrosine kinase inhibitors for the treatment of non-small-cell lung cancer. Onco Targets Ther. 2019;12:5467-5484. doi:10.2147/OTT.S194870

28. Vishwanathan K, Dickinson PA, Bui K, et al. The effect of food or omeprazole on the pharmacokinetics of osimertinib in patients with non-small-cell lung cancer and in healthy volunteers. $J$ Clin Pharmacol. 2018;58(4):474-484. doi:10.1002/jcph.1035

29. van Leeuwen RW, Peric R, Hussaarts KG, et al. Influence of the acidic beverage cola on the absorption of erlotinib in patients with non-small-cell lung cancer. J Clin Oncol. 2016;34(12):1309-1314. doi:10.1200/JCO.2015.65.2560

\section{Publish your work in this journal}

Cancer Management and Research is an international, peer-reviewed open access journal focusing on cancer research and the optimal use of preventative and integrated treatment interventions to achieve improved outcomes, enhanced survival and quality of life for the cancer patient.
The manuscript management system is completely online and includes a very quick and fair peer-review system, which is all easy to use. Visit http://www.dovepress.com/testimonials.php to read real quotes from published authors. 